\title{
Operational Cost Reduction in WDM Networks using Lighttours
}

\author{
Jose L. Marzo", Luis F. Caro", Fernando Solano*, Jaudelice C. de Oliveira ${ }^{\dagger}$, Ramon Fabregat" \\ * University of Girona, Girona, Spain \\ ${ }^{\dagger}$ Drexel University, Philadelphia, USA
}

\begin{abstract}
Wavelength Division Multiplexing (WDM) networks have been adopted as a near-future solution for the broadband Internet. In previous work we proposed a new architecture, named Enhanced Grooming $\left(G^{+}\right)$, that extends the capabilities of traditional optical routes (lightpaths). In this paper, we compare the operational expenditures incurred by routing a set of demands using lightpaths with that of lighttours. The comparison is done by solving an Integer Linear Programming (ILP) problem based on a path formulation. Results show that, under the assumption of single-hop routing, almost $15 \%$ of the operational cost can be reduced with our architecture. In multi-hop routing the operation cost is reduced in $7.1 \%$ and at the same time the ratio of operational cost to number of Optical-Electro-Optical conversions is reduced for our architecture. This means that ISPs could provide the same satisfaction in terms of delay to the end-user with a lower investment in the network architecture.
\end{abstract}

Keywords: lighttours, WDM, traffic grooming, operational cost, lambda-monitoring.

\section{INTRODUCTION}

Wavelength-Division-Multiplexing (WDM) technology has increased network capacity to unprecedent limits in order to satisfy the increasing requirements of the Internet. A (WDM) network consists of a set of interconnected Wavelength-Routing Switches (WRS) that route traffic demands by means of I. A lightpath is a wavelength circuit that connects a pair of WRS in order to transmit data all-optically between them.

Most demands require sub-wavelength bandwidth. If each demand is routed through a unique lightpath, there will be unallocated bandwidth. In order to prevent this, several demands can be multiplexed in the same wavelength. This is denominated Traffic Grooming [1]. When traffic grooming is performed, a lightpath can forward several demands together and a demand can be forwarded by different consecutive lightpaths. However, to be able to do that, demands need to be multiplexed in the electronic domain. Therefore incurring in conversions between the optical and electronic domain that increase the traffic delay.

In [2] a new architecture for performing traffic grooming, named Enhanced Grooming ( $\left.G^{+}\right)$, is proposed. $G^{+}$, allows a transparent WRS to aggregate more traffic over optical routes (lighttours) without signal regeneration. The performance of the new architecture is evaluated using a 0-1 Integer Lineal Programming (ILP) link-based model that solves the TRAFFIC GRoOMIng, Routing AND WAVELENGTH AssignMENT (GRWA) $[3,4,5,6]$ problem. The model studies the network throughput and the number of OEO conversions made. An heuristic is also proposed.

In this paper the operating cost of performing $G^{+}$and classical grooming is studied by analyzing the prices of the components that each node uses to route a given set of demands. This is done by using a new path-based ILP model that takes into account the components market prices and minimizes the total cost. Due to the complexity of the model, the network is assumed to have full wavelength conversion.

\section{NETWORK ARCHITECTURES}

In this section both architectures considered in this paper are described. The WRS functionality can be classified as:

- Send Traffic performing electro-optical conversion (EO): the node has Traffic queued electronically (from itself or from a dropped optical route) and needs to transmit it.

- Receive Traffic performing optical-electro conversion (OE): the node has Traffic that needs to be converted from optical to electrical because it is the destination or it has to be forwarded using another optical route.

- Forward Traffic all optically (OO): an optical path that does not carry traffic needs to be buffered electronically, so the node just has to switch the optical route from one port to another.

\section{A. The Classical Lightpaths}

Lightpaths are end-to-end optical connections established between any pair of WRSs in an optical network. In order to allow a large set of different lightpath configurations in a network, a typical WRS architecture must both forward optically lightpath traffics to other WRS without making OEO conversions and, transmit (or receive) optical traffic over (or from)a lightpath. For more details about a classical WRS the reader is referred to [7].

With the support of the Department of Universities, Research and Information Society (DURSI) of the Government of Catalonia and the European Social Funds (2005-SGR-00296, 2006-FI-00112 and, 2006-FIR-00109). Also with the support of the Spanish Science and Technology Ministry under contract TIC2003-05567 and, the COST project action number 293: Graphs \& Algorithms in Communication Networks. 
In order to route traffic, a classical WRS works as follows: For the EO case traffic queued electronically is retransmitted over a wavelength using a tunable transmitter $(T)$ then it enters the Photonic Cross Connect (PXC) that routes it to the corresponding output wavelength port where it is multiplexed (by the mux) and finally sent through the fiber. For the OE case the lightpath comes from an input port and it is demultiplexed (by the demux). Then the wavelength carrying the lightpath enters the PXC where is redirected to a set of receivers (R) that convert it to the electronic domain and store it in the Electronic Switch for electronic processing. Finally for the OO case the lightpath comes from an input port and it is demultiplexed (by the demux). Then the wavelength carrying the lightpath enters the PXC where is redirected to the corresponding output port where it is multiplexed (by the mux) and finally sent through the fiber.

\section{B. $G^{+}$Network architecture}

The $G^{+}$architecture is depicted in Figure 1. The one presented in [2] has been slightly adjusted due to the market availability of the node components. Nevertheless both architectures have the same functionality.

This architecture works under the following assumptions: a) the net-work transmits optical packets in fixed time-slots, b) packets are optically coded with a fixed length, c) higher layers are able to segment and reassemble optical packets and, $d$ ) the tuning times of the transmitter are smaller than the slot duration.

The main difference between our architecture $\left(G^{+}\right)$and the classical lightpaths architecture is that a WRS node can send electronic traffic not only by creating a lighttour but also by adding traffic on a forwarded lighttour.

In order to route traffic $G^{+}$node works as follows:

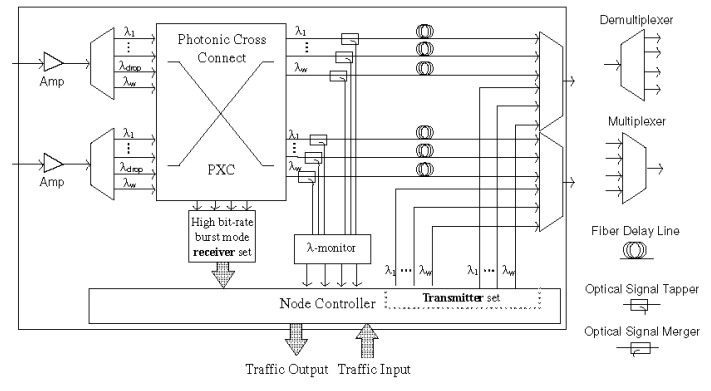

Fig. 1. $G^{+}$WRS architecture.

For the OO case the lighttour comes from an input port and it is demultiplexed (by the demux). Then the wavelength enters the PXC where is redirected to the corresponding output port. Then, it is tapped and a fraction of its light is redirected to the $\lambda$-monitor device. It enters the fiber delay line, then it is merged and multiplexed with the traffic sent by the transmitter set in EO (in case it was sent) using the mux. For the EO case the node, based on the information the $\lambda$-monitor device and information traffic queued electronically, selects a free (without a lighttour or with one that has a free slot) port and wavelength, then the traffic is retransmitted over it using a tunable transmitter set. Then it enters the Multiplexers of the corresponding output port where it is multiplexed and merged with the forwarded lighttour (if any) and finally sent through the fiber. Finally for the $\mathrm{OE}$ case is the same as in the classical lightpaths architecture.

\section{OPERATIONAL COST MODEL}

In this section, an ILP model for the multilayer problem aiming at minimizing operational costs is proposed.

\section{A. Index, Parameters and Variables}

The following list are the indices and parameters and variables used in the ILP proposed.

INDICES:

$i, j \quad$ WRSs in the network.

$m \quad$ A demand that needs to be routed.

$t \quad$ A lighttour in the network.

VARIABLES:

$\rho^{m, t} \quad 1$ if lighttour $t$ routes demand $\mathrm{m}, 0$ otherwise.

$d_{j}^{t} \quad 1$ if WRS $j$ is grooming additional traffic into lighttour $t, 0$ otherwise.

$p^{t} \quad 1$ if lighttour $t$ routes a demand, 0 otherwise
PARAMETERS:

$F_{(i, j)} \quad$ The physical (fiber) distance between nodes. A disconnected pair of nodes has distance 0 .

$L_{t, i, j} \quad 1$ if lighttour $t$ uses the physical link $(i, j)$ and 0 otherwise.

$H \quad$ The maximum number of virtual hops.

\section{B. Operational Cost Functions}

A list of some component market prices can be appreciated in Table 1 and the components unit price (per wavelength) with their respective symbol used in the ILP model are also shown in Table 2.

The operating cost of a network equipped for performing $G^{+}$is calculated. The component prices are based on the number of wavelengths they handle and a cost function which is estimated for each node based on the components that the node uses in order to route the demands.

We define the cost of the extra components that are needed only in a $G^{+}$node as: 


$$
\kappa_{G^{+}}=\kappa_{\lambda}+\Delta \cdot \kappa_{F}+\kappa_{M}
$$

Where $\Delta$ is the distance in kilometres that takes the Fiber Delay line. The Cost function for a $G^{+}$node is defined as follows:

$$
C(j)=\left(\kappa_{T}+\kappa_{G^{+}}\right) \cdot \underbrace{\sum_{t} d_{j}^{t}}_{\alpha}+\kappa_{R} \cdot \underbrace{\sum_{t} E_{j}^{t} \cdot p^{t}}_{\beta}+\left(\kappa_{D}+\kappa_{X}\right) \cdot \underbrace{\sum_{i, t} L_{t, i, j} \cdot p^{t}}_{\gamma}+\kappa_{M} \cdot \underbrace{\sum_{i, t} L_{t, j, i} \cdot p^{t}}_{\delta}+\kappa_{F} \cdot \underbrace{\sum_{i, t} L_{t, j, i} \cdot F_{(j, i)} \cdot p^{t}}_{\rho}
$$

Where $\alpha$ represents total number of lighttours that node $j$ inserts traffic to covering case EO. $\beta$ represents the total number of lighttours that end at node $\mathrm{j}$ covering case $\mathrm{OE} . \gamma$ represents the total number of incoming lighttours at node $j$ covering cases $\mathrm{OE}$ and $\mathrm{OO} . \delta$ represents the total number of outgoing lighttours at node $j$ covering cases $\mathrm{EO}$ and $\mathrm{OO}$. Finally $\rho$ represents the used fiber covering all cases.

The objective function of the ILP model is to minimize the Network Operational Cost, this is expressed in Equation 2 which consist of the sum of the operational cost of all the nodes in the network.

MINIMIZE:

$$
\sum_{j} C(j)
$$

\section{Model Constraints}

The model has all the traditional routing and wavelength assignment constraints, such as the flow conservation constraints, routing constraints, bandwidth constraints, wavelength constraints and a constraints that forces $100 \%$ throughput. It also has the following constraint.

$$
\sum_{t} \rho^{m, t} \leq H, \forall m
$$

Equation 3 limits the number of maximum lighttours used to route each demand to $H$ which restricts the model to perform only singlehop grooming when $H=1$. In order to model multihop the constraint is removed.

a) Classical grooming Model: For classical grooming modeling, the ILP can be restricted by limiting every node to only groom additional traffic into lighttours that begin at the node. Also the node cost function of Equationl must be replaced by:

$$
C(j)=\left(\kappa_{T}+\kappa_{X}\right) \cdot \underbrace{\sum_{i} d_{j}^{t}}_{\alpha}+\kappa_{R} \cdot \underbrace{\sum_{t} E_{j}^{t} \cdot p^{t}}_{\beta}+\left(\kappa_{D}+\kappa_{X}\right) \cdot \underbrace{\sum_{i, t} L_{t, i, j} \cdot p^{t}}_{\gamma}+\kappa_{M} \cdot \underbrace{\sum_{i, t} L_{t, j, i} \cdot p^{t}}_{\delta}+\kappa_{F} \cdot \underbrace{\sum_{i, t} L_{t, j, i} \cdot F_{(j, i)} \cdot p^{t}}_{\rho}
$$

Table 1. Components market prices.

\begin{tabular}{|l||l|}
\hline Component & Market Price \\
\hline Multiplexer $(40 \mathrm{wv})$ & US $\$ 7,000$ \\
\hline Demultiplexer $(40 \mathrm{wv})$ & US $\$ 7,000$ \\
\hline $\begin{array}{l}\text { OXC (32 ports } ~ 160 \\
\text { ports) }\end{array}$ & US $\$ 53,000$ \\
\hline$\lambda$-monitor $(1 \mathrm{wv})$ & US $\$ 210,000$ \\
\hline Fiber $(1 \mathrm{~km})$ & US $\$ 162$ \\
\hline Optical signal merger & US $\$ 612.5$ \\
\hline Optical signal tapper & (1 demux used instead) \\
\hline Transmitter $(1 \mathrm{wv})$ & US $\$ 15,500$ \\
\hline Receiver $(1 \mathrm{wv})$ & US $\$ 350$ \\
\hline
\end{tabular}

Table 2. Components unit prices and symbols.

\begin{tabular}{|l|l||l|}
\hline Symbol & Component & unit Price \\
\hline$\kappa_{M}$ & Multiplexer & US\$175 X wv \\
\hline$\kappa_{D}$ & Demultiplexer & US\$175 X wv \\
\hline$\kappa_{X}$ & $\begin{array}{l}\text { Optical Cross- } \\
\text { Connect }\end{array}$ & $\begin{array}{l}\text { US\$1656.25 X } \\
\text { port }\end{array}$ \\
\hline$\kappa_{\lambda}$ & $\lambda$-monitor & US\$162 X wv \\
\hline$\kappa_{F}$ & Fiber & US\$612.5 X km \\
\hline$\kappa_{T}$ & Transmitter & US\$15,500 X wv \\
\hline$\kappa_{R}$ & Receiver & US\$350 X wv \\
\hline
\end{tabular}

Note that Equation 4 is the same as Equation 1 but with $\kappa_{G^{+}}=\kappa_{X}$, this is because the classical grooming node doesn't use the components that are evaluated in, but in the $\mathrm{OE}$ uses the PXC when the $G^{+}$node does not so $\kappa_{X}$ must replace $\kappa_{G^{+}}$.

\section{SIMULATION RESULTS}

Since the complexity of solving ILP models is high [8], the simulations are performed over the same small topology used for the ILP model presented in [2] and the branch and bound process is interrupted after few iterations, (as many other contributions do) obtaining very good results. It is considered that each fiber link maybe demultiplexed to at most 5 wavelengths and that the capacity of each wavelength is OC-48. Every WRS in the network has 5 transmitters and5 receivers. A set of 87 randomly generated demands are considered as input to the problem. The selection of the source-destination WRS and bandwidth (which may correspond to 
OC-1, OC-3 or OC-12) of each demand, is done randomly using a uniform distribution. The total requested bandwidth is equivalent to OC-290.

In this section numerical examples minimizing the total network operational cost to route all traffic demands for singlehop $(H=1)$, 3-hop-max $(H=3)$ and multihop grooming are presented. The results are summarized on Table 3.

Table 3. Comparison between optimal solutions using $G$ and $G^{+}$.

\begin{tabular}{l|c|c|c|c|c|c|c|c|c}
\hline Hops & \multicolumn{3}{|c|}{ MultiHop } & \multicolumn{3}{c|}{ 3-hop-max } & \multicolumn{3}{c}{ Single Hop } \\
\hline Grooming & $G$ & $G^{+}$ & Diff & $G$ & $G^{+}$ & Diff & $G$ & $G^{+}$ & Diff \\
\hline Cost. & 252 & 234 & $7.1 \%$ & 260 & 244 & $6.15 \%$ & 565 & 487 & $13.8 \%$ \\
\hline OEO Conversions. & 78 & 72 & 6 & 69 & 64 & 5 & 0 & 0 & 0 \\
\hline 1-hop routing. & 36 & 36 & 0 & 39 & 39 & 0 & 87 & 87 & 0 \\
\hline 2-hops routing. & 31 & 36 & -5 & 29 & 34 & -5 & 0 & 0 & 0 \\
\hline$>$ 2-hops routing. & 21 & 16 & 5 & 20 & 15 & 5 & 0 & 0 & 0 \\
\hline
\end{tabular}

The simulations show that for single-hop routing there are considerable savings in terms of operational expenditures: almost USD80.000(13.8\% of the classical architecture). When multi-hop routing is allowed in the network, $G^{+}$shows savings in terms of operational expenditures: USD 16.000 and 3-hop-max shows USD 18.000. Although the difference can be considered small, in both cases the number of OEO conversions needed to route all the traffic is reduced considerably(6 OEO Conv. less in Multihop and 5 in 3-hop-max) regards to the classical architecture. This is a mayor breakthrough since it means that $G^{+}$can route demands with lesser delay (OEO Conversions) and lesser cost than classical grooming.

Comparing single-hop and multi-hop solutions to the problem, it can be said that the ratio of operational cost to number of OEO conversions is smaller for our architecture. This means that ISPs could provide the same satisfaction in terms of delay to the end-user with a lower investment in the network architecture.

\section{CONCLUSIONS}

In this paper, we outline the savings in operational expenditures incurred by using a new architecture, named Enhanced Grooming $\left(G^{+}\right)$, proposed in our previous work. The previously proposed architecture is briefly described and compared with the classical grooming architecture by highlighting the optical components used by each. The market prices for the optical components used were sought.

An Integer Linear Programming (ILP) model using a path-based formulation was proposed. The ILP model solves the well-known Grooming, Routing and Wavelength Assignment problem(GRWA) in a physical network with the goal to minimizing the operational cost of the total network.

The simulation results show that $G^{+}$always have less expenditures than classical grooming. The fact is supported since all the solutions using the classical grooming architecture are also found using the $G^{+}$ architecture. Most operators aim at using single-hop routing in WDM networks. In this case, the revenues are around $15 \%$ with our architecture.

\section{REFERENCES}

[1] E. Modiano and P. J. Lin, "Traffic grooming in WDM networks," IEEE Commun. Mag., vol. 39, no. 7, pp. 124-129, Jul. 2001.

[2] F. Solano, L. Caro, J. de Oliveira, R. Fabregat, and J. Marzo, " $\mathrm{G}^{+}$: Enhanced traffic grooming in WDM mesh networks using Lighttours," IEEE J. Sel. Areas Commun., Jun. 2007, to appear. Available online for reviewers at http://bcds.udg.es/papers/solano-jsac-2007.pdf.

[3] H. Zang, J. Jue, and B. Mukherjeee, "A review of routing and wavelength assignment approaches for wavelength-routed optical WDM networks," Optical Networks Magazine, Jan. 2000. [Online]. Available: citeseer.ist.psu.edu/zang00review.html

[4] D. Banerjee and B. Mukherjee, "Wavelength-routed optical networks: Linear formulation, resource budgeting tradeoffs, and a reconfiguration study, "IEEE/ACM Trans. Netw., vol. 8, no. 5, pp. 598-607, Oct. 2000.

[5] C.Q. Chunsheng Xin and S. Dixit, "Traffic grooming in mesh WDM optical networks performance analysis," IEEE J. Sel. Areas Commun., vol. 22, no. 9, pp. 1658-1669, Nov. 2004.

[6] W.Yao and B. Ramamurthy, "A link bundled auxiliary graph model for constrained dynamic traffic grooming in WDM mesh networks," IEEE J. Sel. Areas Commun., vol. 23, no. 8, pp. 1542-1555, Aug. 2005.

[7] H. Z.Keyao Zhu and B. Mukherjee, "A comprehensive study on next-generation optical grooming switches," IEEE J. Sel. Areas Commun., vol. 21, no. 7, pp. 1173-1186, Sep. 2003.

[8] D.P. Bertsekas, Network Optimization: Continuous and Discrete Models. Athena Scientific, 1998. 